\section{More than skin deep}

\section{By Lauren Martz, Staff Writer}

Researchers at the Medical College of Wisconsin have developed a way to engineer botulinum toxins for indications beyond neuromuscular conditions. ${ }^{1}$ The new compounds offer oral bioavailability and selectivity for non-neuronal drug targets, although immunogenicity and toxicity concerns must still be addressed.

Botulinum toxins are extremely potent blockers of neurotransmission, which has made them attractive therapeutics at low doses for neuromuscular conditions such as strabismus, blepharospam, hemifacial spasm and cervical dystonia, as well as cosmetic use. ${ }^{2}$

The toxins, produced by Clostridium botulinum, naturally block nerve signaling by inhibiting the release of the contents of synaptic vesicles. The toxin cleaves neuronal soluble $N$ -
"In terms of indications, this could be targeted for nervous disorders and respiratory conditions such as hyper-secretion of mucus."

\section{-Jacques-Pierre Moreau,} Ipsen Group protein can now cleave a non-neuronal SNARE protein, which could inhibit diseases that are mediated by hyper-exocytosis," said corresponding author Joseph Barbieri. Barbieri is professor of microbiology and molecular genetics and director of the medical scientist teaching program at the Medical College of Wisconsin.

\section{Expanded potential}

Barbieri told SciBX that the next step is to engineer the modified toxin light chains to bind a cell-specific receptor to determine how efficiently and specifically the toxin can be targeted.

"Botulinum toxin is very unique because it serves as a good template: it is orally active, extremely selective and is active for a long time. These properties make it an interesting platform for modification," noted Moreau.

Ipsen markets Dysport abobotulinumtoxin A to treat motor disorders and muscle spasticity, and for aesthetic applications. The botulinum neurotoxin A complex is also approved to treat cervical dystonia and glabellar lines. Galderma S.A. and Medicis Pharmaceutical Corp. market the drug for the aesthetic uses.

Another potential use for the engineered toxins is "for regulation of hyper-secretion diseases or inflammatory problems. Clearly, ethylmaleimide-sensitive fusion protein-attachment protein receptors (SNAREs), such as synaptosomal-associated protein 25 (SNAP25), at the neuromuscular junction to prevent vesicle binding to the plasma membrane and thus impede the release of neurotransmitters from the vesicle. $^{3}$

The toxin has three components: a light chain that controls catalytic activity, a heavy chain that controls cellular binding to neurons and a translocation domain that facilitates toxin movement across the plasma membrane.

Jacques-Pierre Moreau, EVP and CSO of Ipsen Group, told SciBX that other groups have modified the heavy chain in order to target receptors on non-neuronal cells. The problem is that retargeting the toxins appears to also require modification of the catalytic site to shift specificity to non-neuronal ligands.

In a paper published in the Proceedings of the National Academy of Sciences, the Medical College of Wisconsin team reported doing just that-modifying the toxin's light chain. As a result, the toxin's specificity was shifted from neuronal to non-neuronal SNAREs. The result could be therapeutics for non-neuronal secretory diseases.

In vitro, a mutation to botulinum toxin's catalytic site, which naturally targets SNAP25, altered its specificity to the non-neuronal isoform SNAP23, which is involved in processes including fusion reactions in cell growth, membrane repair and synaptic transmission. ${ }^{4}$

The mutated toxins cleaved endogenous SNAP23 and inhibited tumor necrosis factor- $\alpha$ (TNF- $\alpha$ )-mediated secretion of mucin and IL- 8 , which are associated with some secretory diseases.

"The benefit of the engineered light chain is that the modified asthma is a good target," according to Kim Janda, professor in the Department of Chemistry and Department of Immunology and director of the Worm Institute of Research and Medicine at The Scripps

\section{Research Institute.}

"In terms of indications, this could be targeted for nervous disorders and respiratory conditions such as hyper-secretion of mucus. It could also help treat hyperinsulinemia in metabolic syndromes and could control secretion of growth hormones in oncology," said Moreau.

He also thinks the findings described in the PNAS article could have utility in the neurology space. "The ability to modify and optimize the pharmacokinetics could still bring better botulinum toxins to the market. This could lead drug companies to develop toxins better suited for neuromuscular disease," Moreau said.

Barbieri, however, thinks the starting place for the engineered proteins should be in non-neuronal conditions.

"Application of the modified light chains in neurological disorders is limited since the modified light chain is less efficient in cleaving SNAP25, the neural SNARE protein, compared with native botulinum toxin," he said.

\section{Toxicity profile}

Regardless of indication, a key question is whether modifications to the botulinum toxin will alter the molecule's safety profile.

"As you move away from the natural botulinum toxin, you might run into immunogenicity and this must be quantified," said Moreau. "What we learn from botulinum toxin administration is that some people develop antibodies very quickly and will not benefit from 
botulinum toxin therapeutics, while others will not develop antibodies when on treatment for years. Engineering the toxins could lead to immunity development even in people who do not have an immune response to the natural toxin."

Moreau added: "In the engineering, you need to be sure that you maintain superb specificity" for the target protein such as SNAP23. "The last thing you want is crossing over into activity on other organs or tissues. The very nature of blocking secretions is so potent and powerful that it has the potential for very bad toxicity," he said.

Barbieri, however, thinks immunogenicity is unlikely to be an issue. He said a targeting molecule will deliver the modified light chain directly into non-neuronal cells, thus keeping the engineered toxins out of sight of the immune system.

Barbieri told SciBX that a patent application has been filed for the mutated botulinum toxin light chains and the IP is available for licensing.
Martz, L. SciBX 2(24); doi:10.1038/scibx.2009.965

Published online June 18, 2009

\section{REFERENCES}

1. Chen, S. \& Barbieri, J. Proc. Natl. Acad. Sci. USA; published online June 1, 2009; doi:10.1073/pnas.0903111106

Contact: Joseph Barbieri, Medical College of Wisconsin, Milwaukee, Wis.

e-mail: jtb01@mcw.edu

2. Glogau, R. Clin. J. Pain 18, S191-S197 (2002)

3. Montecucco, C. \& Shiavo, G. Mol. Microbiol. 13, 1-8 (1994)

4. Jahn, R. \& Scheller, R. Nat. Rev. Mol. Cell Biol. 7, 631-643 (2006)

\section{COMPANIES AND INSTITUTIONS MENTIONED}

Galderma S.A., Lausanne, Switzerland Ipsen Group (Euronext:IPN), Paris, France

Medical College of Wisconsin, Milwaukee, Wis. Medicis Pharmaceutical Corp. (NYSE:MRX), Scottsdale, Ariz. The Scripps Research Institute, La Jolla, Calif. 